Research Paper

\title{
Integrative Oncology Physician Consultations at a Comprehensive Cancer Center: Analysis of Demographic, Clinical and Patient Reported Outcomes
}

\author{
Gabriel Lopez ${ }^{1 *}$, Jennifer McQuade ${ }^{2 *}$, Lorenzo Cohen ${ }^{\circledR}$, Jane T Williams ${ }^{1}$, Amy R Spelman³, Bryan \\ Fellman', Yisheng $\mathrm{Li}^{4}$, Eduardo Bruera ${ }^{1}$, Richard T Lee ${ }^{5}$ \\ 1. Department of Palliative, Rehabilitation, and Integrative Medicine, The University of Texas MD Anderson Cancer Center, Houston, TX; \\ 2. Melanoma Medical Oncology, The University of Texas MD Anderson Cancer Center, Houston, TX; \\ 3. Department of Radiation Oncology, The University of Texas MD Anderson Cancer Center, Houston, TX; \\ 4. Department of Biostatistics, The University of Texas MD Anderson Cancer Center, Houston, TX; \\ 5. Division of Hematology and Oncology, Case Western Reserve, Cleveland, $\mathrm{OH}$. \\ *Contributed equally and both considered first authors.
}

$\triangle$ Corresponding author: Lorenzo Cohen, PhD, Professor, Department of Palliative, Rehabilitation and Integrative Medicine, The University of Texas MD Anderson Cancer Center, 1515 Holcombe Blvd, Unit 1414, Houston, TX, 77030; T: (713) 745-4260; F: (713) 745-2437.

(C) Ivyspring International Publisher. This is an open access article distributed under the terms of the Creative Commons Attribution (CC BY-NC) license (https://creativecommons.org/licenses/by-nc/4.0/). See http://ivyspring.com/terms for full terms and conditions.

Received: 2016.09.07; Accepted: 2016.10.29; Published: 2017.02.10

\begin{abstract}
Background: Integrative oncology $(\mathrm{IO})$ is a relatively new field that seeks to bring evidence-based, non-conventional approaches into conventional oncology care in a coordinated and safe manner. Though complementary and alternative medicine (CAM) are highly utilized by cancer patients, little is known about the characteristics of patients seeking IO consultation.

Methods: Patients presenting for an outpatient 10 consultation completed a CAM use questionnaire, Measure Yourself Concerns and Wellbeing (MYCaW), Edmonton Symptom Assessment Scale (ESAS), Quality of Life Short Form 12 (SF-12), and post-consultation satisfaction item.

Results: 2,474 new patient IO consultations were conducted from 9/2009 to 12/2013 and 2367 (96\%) completed at least one measure. Most were female (69\%); the most frequent cancer type was breast (29\%); $38 \%$ had distant/advanced disease; 75\% had used a CAM approach in prior 12 months. The most common concerns were seeking an integrative/holistic approach (34\%), herbs/supplements (34\%), and diet/nutrition $(21 \%)$. Overall symptom burden was low, with baseline symptom scores (ESAS) highest (worst) for sleep (4.2; SD 2.8), fatigue (4.0; SD 2.8), and well-being (3.8; SD 2.6). On the SF-12, the physical health scores (35.3; SD 7.5) were significantly lower than that of a healthy population (50), but mental health scores were not (46.8; SD 10.2). Satisfaction was high (9.4; SD 1.3) with the consultation.

Conclusions: Patients presenting for 10 consultation tended to have early stage disease, had previously used a CAM approach, had a relatively low symptom burden, and were most interested in developing an integrative approach to their care or discussing herbs/supplement use.
\end{abstract}

Key words: Integrative Medicine, Integrative Oncology, Complementary Medicine, Patient Reported Outcomes, Symptom Assessment.

\section{Introduction}

An estimated $30-50 \%$ of cancer patients use complementary or alternative medicine [1,2]. Though some patients pursue these treatments under the guidance of a physician, patients more often turn to the internet, lay press, friends and family, or alternative practitioners to inform their approach [3]. 
Risks to this unsupervised approach include eschewing proven therapies in favor of untested interventions, potential for direct organ toxicity from unproven therapies, and/or negative impact on treatment efficacy as a result of herb/supplement-drug interactions.

Integrative medicine (IM) is a discipline that seeks to bring evidence-based, non-conventional approaches into conventional medical care in a coordinated and safe manner. IM is increasingly becoming a part of health care services at academic centers across the United States and internationally, with over 62 member institutions forming the Academic Consortium for Integrative Medicine and Health in the US [4], Canada and Mexico. Given the unique needs of cancer patients, most major National Cancer Institute (NCI) designated comprehensive cancer centers now have an IM program, although available services vary widely [5]. The term integrative oncology (IO) is used to describe the application of integrative medicine to the care of cancer patients.

The University of Texas MD Anderson Cancer Center's Integrative Medicine Program was established in 1998. The clinical center offers group programs as well as individual services including oncology massage, acupuncture, physical therapy, nutrition, meditation, health psychology, and music therapy. Our clinical model [Figure 1] is based on the bio-psychosocial model of care, first espoused by George Engel, seeking to incorporate therapies that address physical, psycho-spiritual, and social dimensions of health [6].

While some services can be referred to directly by providers within the institution (acupuncture, oncology massage, music therapy, meditation), the vast majority of patients are initially referred to and seen in consultation by an IO physician. The goal of the physician consultation is to provide patients with an integrative care plan tailored to the individual and his/her unique disease trajectory. This plan may include referrals to services such as acupuncture or massage for symptom control; health psychology, meditation, or music therapy for psychological distress; counseling on healthy lifestyle behaviors and referral to nutrition and physical therapy; or discussion of risk and evidence-base for herb/supplements or alternative treatments being pursued or considered by patients.

\section{Physical}

- Exercise

- Nutrition

- Acupuncture

- Oncology Massage

- Primary Oncology team

- Physical Medicine \& Rehabilitation

- Supportive Care

- Cancer Pain

\section{Mind-Body}

- Health Psychology

- Meditation

- Music Therapy

- Tai Chi/Qi Gong

- Yoga

- Psychiatry

- Spiritual Care

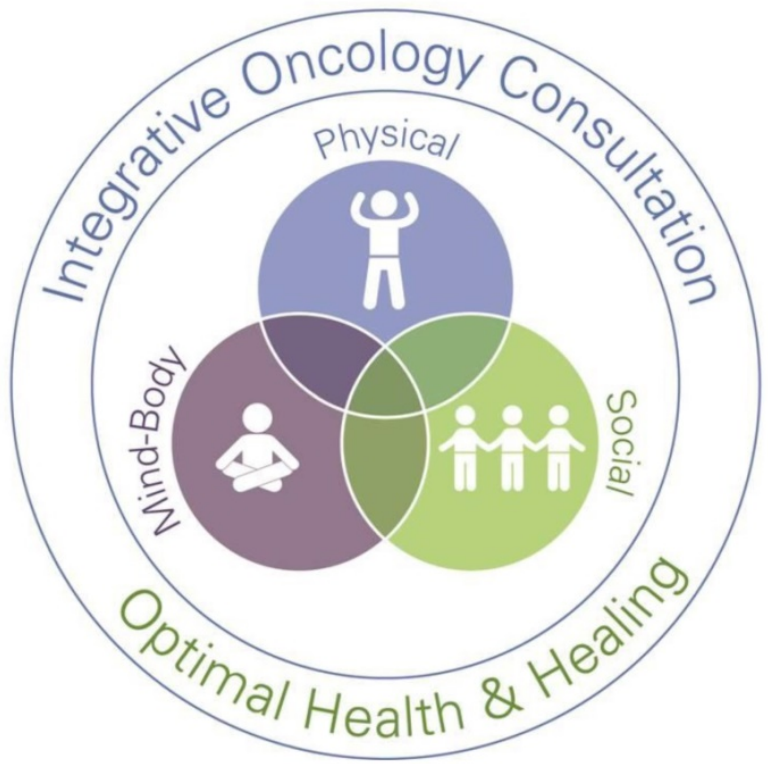

\section{Social}

- Group Programs

- Social Work

- Support Groups

Figure 1: Integrative medicine center model. Services and group classes offered by center in bold. 
While the characteristics of patients that pursue complementary and alternative medicine have been well-studied [1,2], there is little published data on the new but rapidly growing provision of $\mathrm{IO}$ consultations $[3,7,8]$. To address this lack of knowledge, we examined the demographics, disease characteristics, reason for consultation, symptom burden, and satisfaction, of an IO consultation at a major US cancer center.

\section{Methods}

This study was conducted at the University of Texas MD Anderson Cancer Center's Integrative Medicine Center between September 2009 and December 2013. All patients presenting for an IO physician consultation were asked to complete a series of questionnaires as part of an IRB approved protocol; only patients age $>18$ were included in this analysis. Immediately prior to their physician consultation, patients completed a complementary and alternative medicine (CAM) use questionnaire, the Measure Yourself Concerns and Wellbeing (MYCaW), Edmonton Symptom Assessment Scale (ESAS), and Medical Outcomes Study Short Form 12 (SF-12). Immediately after the physician consultation, patients were asked how well their top two MYCaW concerns were addressed as well as a single question regarding overall satisfaction with the encounter.

\section{Intervention}

The IO consultation includes a comprehensive assessment, responding to both patient self-reported data made available to the clinician immediately prior to the encounter and patient concerns elicited through narrative during the face-to-face encounter. As part of the IO consultation, the majority of patients meet with an advanced practice provider immediately prior to meeting with the physician. Patient interest in and use of complementary and alternative medicine is explored and a complete history and physical are conducted. Expectations of integrative medicine are elicited, as are bio-psychosocial concerns. The approach of the MD Anderson integrative medicine center is introduced; specifically, the bio-psychosocial model [6] and evidence-based approach. The risks and benefits of specific modalities of interest to the patient are discussed. Specifically, the literature is reviewed for any high-quality clinical evidence of benefit, interactions with conventional therapies, and potential toxicities. Specific modalities that may benefit the patient's symptoms are introduced and recommendations are given for healthy lifestyle behaviors, with a focus on diet, exercise, and stress management when appropriate. Individual services to which patients may be referred within the center include oncology massage, acupuncture, physical therapy, nutrition, meditation consultation, health psychology, and music therapy; referrals are also made to group classes including yoga, qi-gong/tai-chi, and Tibetan meditation. The consultation is both an education session and opportunity to identify symptoms that may benefit from an integrative approach.

\section{Measures}

All instruments were completed using paper forms and then entered into an electronic database for analysis. Patient demographics and clinical data were extracted from the medical record. Our internally developed CAM use questionnaire asked patients about CAM use in the year preceding their consultation; CAM items were based on the NIH-National Center for Complementary and Integrative Health $(\mathrm{NCCIH})$ complementary health categories of natural products (herbs, vitamins), mind and body approaches (acupuncture, qi-gong/tai-chi, guided imagery/meditation, relaxation techniques, hypnosis, massage, chiropractic care, music therapy, yoga, pilates), and other complementary health approaches (e.g., homeopathy). This questionnaire was administered for consultations from Jan 1, 2013 through Dec 31, 2013.

\section{Measure Yourself Concerns and Wellbeing (MYCaW)}

Patients completed a modified version of the MYCaW questionnaire [9]. Patients identified the top two concerns for their integrative medicine consultation from a list of available topic areas including integrative/holistic approach, herb/supplements, diet/nutrition, pain, overall health and stress/anxiety, as well as an "other" category. In addition, patients were asked to rate the importance of each concern on a scale from $0-10$, with 10 being highly important.

\section{Edmonton Symptom Assessment Scale (ESAS)}

Patient symptom burden was assessed using the ESAS [10]. Patients were asked to report on ten symptoms: pain, fatigue, nausea, depression, anxiety, drowsiness, loss of appetite, decreased sense of well-being, shortness of breath, and sleep-as experienced in the prior 24 hours on a numeric scale of 0 to 10, in which 10 is the worst possible expression of that symptom. The global distress score is the sum of pain, fatigue, nausea, drowsiness, appetite, shortness of breath, anxiety, depression, and well-being scores. The physical distress score is the sum of pain, fatigue, nausea, drowsiness, appetite, 
and shortness of breath. The psychological distress score is the sum of anxiety and depression.

\section{Quality of Life (QOL)}

QOL was measured using the Medical Outcomes Study Short Form 12 (SF-12) for patients presenting between June 17, 2010 through April 18, 2013. The SF-12 is a general 12-item QOL survey instrument; the physical health (PCS) and mental health (MCS) component T-scores were compared to a national norm with a mean score of 50.0 and a standard deviation of 10.0. Half a standard deviation in score, or 5 points, represents a clinically significant difference.

\section{Satisfaction}

Overall satisfaction with the encounter was assessed on a 0 to 10 scale [0-1 very dissatisfied, 2-4 dissatisfied, 5 neutral , 6-8 satisfied, 9-10 very satisfied].

\section{Statistical Analyses}

Summary statistics were used to describe the demographic characteristics of those visiting the IM consult clinic. Summary statistics were calculated for QOL questionnaires and on reasons for coming to clinic. Frequencies of types of visit after the initial IM consult visit were calculated to determine the most frequented service lines used after IM consult visit. Differences in ESAS and MYCaW were also examined based on disease status comparing patients with loco-regional versus distant metastatic disease using a Wilcoxon rank-sum and chi-squared test respectively. P-values of $\leq 0.05$ were considered statistically significant.

\section{Results}

Between September 1, 2009 and December 31, 2013 , a total of 2,474 new patient integrative oncology consultations were conducted; 643 (26\%) had at least one visit following their initial consultation. Two-thousand three hundred and sixty-seven (96\%) patients completed at least one measure and had data available for this analysis [Table 1]. Patients were mostly female $(69 \%)$ and white $(75 \%)$, average age of 56 , with the majority (61.9\%) from the state of Texas. Depending on the outcome assessed, missing data on the full survey ranged from $0-25 \%$, a common level for these types of clinic-based survey studies.

The most frequent cancer types included breast (29.3\%), gastrointestinal (13.1\%), and thoracic/head and neck $(8.4 \%)$ with $38 \%$ of all staged patients having distant/advanced disease. For the CAM questionnaire which was implemented in 2013, data were available for 799 of the 801 initial consultations.
Five hundred and ninety-five patients (74.5\%) had used a CAM approach in the prior 12 months [Figure 2]. Of those participants whom used at least one CAM approach in the prior 12 months, the most common modalities used were vitamins $(75.1 \%)$, massage $(33.4 \%)$, and herbal products $(29.7 \%)$.

Table 1: Demographic characteristics of study sample.

\begin{tabular}{lll}
\hline Characteristic & N & $\%$ \\
\hline Age & & \\
Total patients & 2377 & \\
Mean (SD) & $55.9(12.6)$ & \\
Median (Min-Max) & $57(18.1-90.6)$ & \\
Gender & & \\
Female & 1637 & 68.9 \\
Male & 740 & 31.1 \\
Race & & \\
Black & 170 & 7.2 \\
White & 1780 & 74.9 \\
Spanish Surname & 240 & 10.1 \\
Other & 186 & 7.8 \\
Residence & & \\
Harris County & 706 & 29.7 \\
7 Surrounding Counties & 284 & 11.9 \\
Rest of Texas & 483 & 20.3 \\
Rest of US & 825 & 34.7 \\
International & 79 & 3.3 \\
Disease Type & & \\
Breast & & \\
Gastrointestinal & 696 & 29.3 \\
Genitourinary & 312 & 13.1 \\
Gynecologic & Leukemia \\
Lymphoma/Myeloma & 116 & 4.9 \\
Sarcoma & 144 & 6.1 \\
Skin (including melanoma) & 41 & 1.7 \\
Thoracic/Head and Neck & 125 & 5.3 \\
Multiple & 102 & 4.3 \\
Other & 71 & 3.0 \\
Stage* & 199 & 8.4 \\
Local & 464 & 19.5 \\
Advanced & 107 & 4.5 \\
*1880 patients with staging information available. & \\
& & \\
& 1170.2 \\
& & 37.8 \\
\hline
\end{tabular}

Based on the MYCaW [Figure 3], the most common reasons for presenting to an initial integrative medicine consultation were seeking an integrative approach (34.2\%), questions about herbs/supplements $(33.8 \%)$, and diet/nutrition $(21.1 \%)$. Up to $13 \%$ of patient concerns were for specific symptoms such as pain, stress/anxiety, fatigue, sleep, neuropathy, and hot flashes. Patient interest in discussing their top two concerns was high, with scores of 8.4 (SD 2.1; $\mathrm{n}=2135$ ) and 9.2 (SD 1.8; $\mathrm{n}=1439$ ), respectively. Patients reported high satisfaction with addressing their top two concerns, 8 (SD 2.2; $\mathrm{n}=1989$ ) and 9.1 (SD 1.8; $\mathrm{n}=1368$ ), as well as with the overall clinical encounter 9.4 (SD 1.3; $\mathrm{n}=1575$ ). 


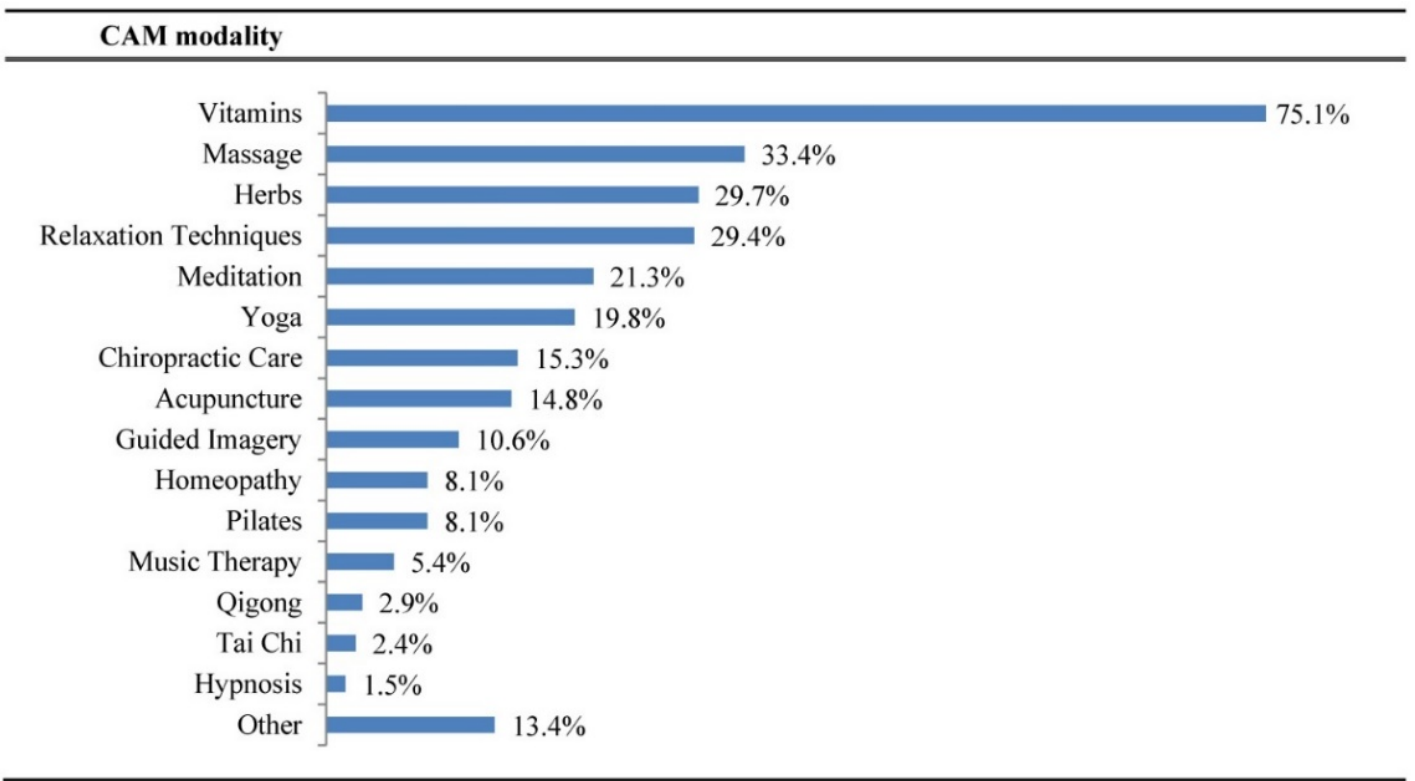

Figure 2: Of those that have used CAM in past 12 months $(n=595)$, which CAMs were used.

\section{MYCaW concerns}

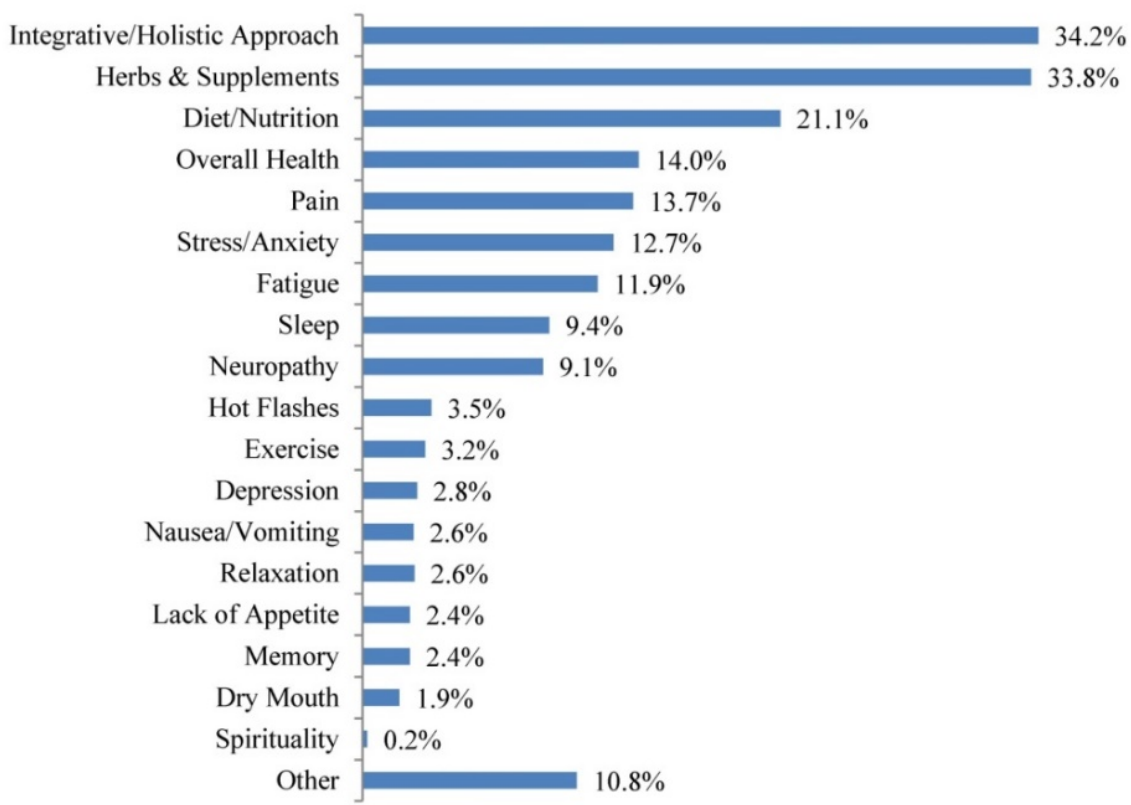

Figure 3: Patient top two concerns for seeking an integrative oncology consultation.

The highest average symptom scores at initial visit as reported using the ESAS [Table 2] were for sleep (4.2), fatigue (4.0), and well-being (3.8); symptoms of fatigue and well-being were the greatest contributors to global distress. For physical symptoms, the highest scores were for sleep (4.2), fatigue (4.0), and appetite (2.8). For psychological symptoms, the mean score was highest for anxiety (3.1) followed by depression (2.2), with anxiety having the greatest contribution to psychological distress.

In measuring QOL using the SF-12 [Table S1], the mean PCS score was 35.3 (range 11.3-61.5; SD 7.5; $\mathrm{n}=1253$ ) and the mean MCS score was 46.8 (range 4.6-76; SD 10.2; $\mathrm{n}=1253$ ). The MCS score approached half a standard deviation below the healthy population mean $(525 / 1253,42 \%$, had a score of 45 or less), but only the physical health score was greater than half a standard deviation below the national mean of 50, and, in fact, approached a 15-point difference (1150/1253, 92\%, had a score of 45 or less), representing a clinically significant differences from the general population. 


\section{Comparison of loco-regional versus distant disease}

The most frequently seen cancer types differed according to disease stage. Breast $(39 \%)$ was most common disease site for those with loco-regional disease and gastrointestinal (25.1\%) for those with distant disease. When comparing mean ESAS scores of patients with loco-regional versus distant disease, those with distant disease had higher baseline symptom distress with statistically significantly higher ESAS scores for pain (2.8 vs 2.4, $\mathrm{p}=0.010$ ), nausea (1.2 vs $0.9, \mathrm{p}<0.001)$, shortness of breath (1.6 vs $1.2, \mathrm{p}<0.001)$, and appetite (3.0 vs $2.6, \mathrm{p}=0.002$ ) [Table S2]. The ESAS subscale scores for physical distress (14.9 vs 13.3, $\mathrm{p}=0.006)$ and global distress (24.1 vs 22.0, $\mathrm{p}=0.020$ ) followed a similar pattern. Regarding QOL, those with loco-regional disease had higher physical health scores (36.1 vs 34; $\mathrm{p}<0.001$ ), and similar values for mental health scores ( 47.3 vs 47.0 ; $\mathrm{p}=0.842$ ). There was no difference in overall satisfaction with IO services. For both groups, developing an integrative/holistic approach was the most common MYCaW interest. Top MYCaW interests for patients with loco-regional disease included herbal products, integrative/holistic approach, and diet; for those with distant disease, the order was integrative/holistic approach, herbal products, and diet. Significantly more patients with distant disease expressed an interest in integrative/holistic approach compared to those with loco-regional disease (38.4\% vs 32.8\%; $\mathrm{p}=0.020)$. Patients with loco-regional disease were more interested in areas of overall health $(16.2 \%$ vs $10.8 \%$, $\mathrm{p}=0.002)$ and hot flashes $(4.6 \%$ vs $1.6 \%, \mathrm{p}=0.001)$ than those with distant disease.

Table 2: ESAS scores for initial consultation.

\begin{tabular}{|c|c|c|c|}
\hline \multicolumn{4}{|c|}{ Initial visit } \\
\hline ESAS & $\mathbf{N}$ & Mean (SD) & (Range) \\
\hline Pain & 2358 & $2.6(2.8)$ & $(0-10)$ \\
\hline Fatigue & 2361 & $4.0(2.8)$ & $(0-10)$ \\
\hline Nauseated & 2344 & $1.0(2.0)$ & $(0-10)$ \\
\hline Sleep & 2316 & $4.2(2.8)$ & $(0-10)$ \\
\hline Shortness of breath & 2332 & $1.4(2.2)$ & $(0-10)$ \\
\hline Appetite & 2353 & $2.8(2.8)$ & $(0-10)$ \\
\hline Drowsy & 2339 & $2.4(2.7)$ & $(0-10)$ \\
\hline Depressed & 2352 & $2.2(2.6)$ & $(0-10)$ \\
\hline Anxious & 2367 & $3.1(2.9)$ & $(0-10)$ \\
\hline Well-being & 2339 & $3.8(2.6)$ & $(0-10)$ \\
\hline Physical Distressa & 2209 & $14(10.5)$ & $(0-58)$ \\
\hline Psychological Distress ${ }^{b}$ & 2340 & $5.3(5.1)$ & $(0-20)$ \\
\hline Global Distressc & 2158 & $23(15.5)$ & $(0-85)$ \\
\hline
\end{tabular}

a) Physical Distress = Pain, Fatigue, Nausea, Drowsiness, Appetite, Shortness of Breath.

b) Psychological Distress = Anxiety, Depression.

c) Global Distress = Pain, Fatigue, Nausea, Drowsiness, Appetite, Shortness of

Breath, Anxiety, Depression, Well Being.

\section{Complementary health service utilization following 10 consultation}

Following the initial IO consultation, a follow up IO visit with the physician and/or advanced practice practitioner was the most common encounter, utilized by 643 patients $(26 \% ; 643 / 2474)$. Six hundred and twenty-four $(25 \% ; 624 / 2474)$ patients accessed one or more of our integrative medicine center services following an IO consultation, for a total of 1098 encounters. Of the 1098 encounters, the services accessed most frequently after the initial physician consultation included acupuncture (52\%), oncology massage (32\%), and physical therapy (5\%). Acupuncture and oncology massage had the highest number of mean visits per participant, being 4.7 and 4 , respectively.

\section{Discussion}

To our knowledge, this study represents the largest, most comprehensive study of patients seen in an integrative oncology consultation. Though the characteristics and motivations of cancer patients who seek complementary and alternative medicine have been well-studied [1,2], little exists in the literature regarding the patients who are seen in a formal integrative oncology consultation. Integrative oncology is a rapidly growing field and understanding the patients served by this service and their needs is key to optimizing the service and designing prospective clinical trials in this field.

Consistent with the published literature on patients who seek complementary and alternative medicine $[1,2]$, the patients seen in our center for an integrative oncology consultation were predominantly women and breast cancer was the most common diagnosis. This is in contrast to the patient population seen by our cancer center's palliative/supportive care service, where the gender is evenly divided and thoracic, head and neck, and gastrointestinal malignancies are more commonly seen than breast cancer [11,12]. Most of our patients had early stage disease, again in contrast to data reported by the MD Anderson palliative/supportive care service where the majority of the patients have advanced disease [11,12].

The three most common patient-reported concerns were developing an integrative approach, herbs/supplements, and diet/nutrition. Although patients reported a wide variety of symptoms, the overall symptom burden was moderate with only fatigue and sleep achieving mean ESAS scores $>4$. Only $9-13 \%$ of patients reported a specific physical (pain, fatigue, neuropathy) or emotional symptom (stress/anxiety) as a primary concern. The patients being seen in the integrative medicine clinic reported 
substantially less symptom burden relative to the levels previously reported by patients seen by the MD Anderson palliative/supportive care service (IO: physical distress $=15$, psychological distress $=5.3$, global distress $=23$; vs. palliative care/supportive care: physical distress $=25.6$, psychological distress $=$ 6.2, global distress $=36.8$ ) [11]. However, as would be expected, the subset of IO patients with distant/advanced disease, did report higher levels of physical and global distress with a lower physical QOL score than those with loco-regional disease.

Rather than being predominantly end-stage patients seeking care after the exhaustion of conventional medical options, most patients were early stage patients presenting to support their overall health during and after cancer treatment. This is in line with the philosophy of integrative oncology, and is in contrast to some of the many misconceptions that integrative oncology should only be considered when all other conventional avenues for "cure" have been exhausted. Although this conception of integrative medicine as analogous with "alternative medicine" is starting to fade, there are still some misconceptions of the field and purpose. It is gratifying to see that the patients being referred are the group of patients most likely to benefit.

The most common integrative oncology modality indicated as a concern for patients was herbs and supplements. Of our population, the majority of patients $(75 \%)$ used a CAM modality in the year preceding their consultation, with vitamins $(75 \%)$ and herbs $(29 \%)$ as the most common modalities. This is consistent with surveys of complementary and alternative medicine use which have consistently found herbs and supplements to be the primary modality used by patients [13-15]. The available evidence of the benefit of herbs and supplements in the treatment of cancer is quite limited, with most of the research still residing in the preclinical or early clinical phase. This means that the use of herbs and supplements comes with unknown, potentially significant risks which can include herb/drug interactions and direct organ toxicity.

The evidence is much stronger for the use of certain integrative modalities for specific cancer related symptoms, such as acupuncture for nausea and vomiting [16] and exercise for fatigue, sleep, anxiety and overall well-being [17]. Thus, patients' interests and concerns are often not aligned with the available evidence. One of the goals of the integrative medicine consultation at our institution is education on the evidence and often involves re-aligning expectations. To address concerns regarding diet and nutrition, patients are counseled based on American Institute for Cancer Research and American Cancer
Society guidelines, which advocate a whole-food, primarily plant-based diet, limiting sedentary behavior, maintaining a healthy body weight, and limiting alcohol consumption [18]. Post consultation MYCaW scores revealed a high level of satisfaction, suggesting patients are finding value in having their top concerns successfully addressed by the IO consultation. Limitations of our study include being conducted at a single institution and thus may not be representative of patients receiving $\mathrm{IO}$ consultations at other comprehensive cancer centers or community oncology settings.

We have demonstrated that by providing consultations on how to bring together conventional and non-conventional treatment approaches in an evidence-based manner, we are providing a service that is valued by patients. Further research should be conducted to understand how this consultation service impacts QOL, symptom burden, and treatment outcomes as well as how it influences patient decision-making and subsequent CAM use.

\section{Supplementary Material}

Tables S1 and S2.

http://www.jcancer.org/v08p0395s1.pdf

\section{Competing Interests}

The authors have declared that no competing interest exists.

\section{References}

1. Gansler T, Kaw C, Crammer C, et al. A population-based study of prevalence of complementary methods use by cancer survivors: A report from the American Cancer Society's studies of cancer survivors. Cancer. 2008;113:1048-1057.

2. Horneber M, Bueschel G, Dennert G, Less D, Ritter E, Zwahlen M. How many cancer patients use complementary and alternative medicine: a systematic review and metaanalysis. Integr Cancer Ther. 2012; 11(3):187-203.

3. Deng G, and Cassileth B. Complementary or alternative medicine in cancer care. Nat. Rev. Clin. Onc. 2013.

4. [Internet] http://www.imconsortium.org/members/members.cfm

5. Seely DM. A systematic review of integrative oncology programs. Current oncology 2012.

6. Engle GL. The need for a new medical model: a challenge for biomedicine. Science 1977; 196: 129-136

7. Frenkel M, Cohen L, Peterson N, et al. Integrative medicine consultation service in a comprehensive cancer center: findings and outcomes. Integr Cancer Ther. 2010 Sep;9(3):276-83

8. Ben-Arye $\mathrm{E}$, et al. Integrating complementary medicine in supportive cancer care models across four continents. Med Oncol. 2013 Jun;30(2):511.

9. Paterson C, et al. Measure Yourself Concerns and Wellbeing (MYCaW): An individualised questionnaire for evaluating outcome in cancer support care that includes complementary therapies. Complementary Therapies in Medicine 2007; 15:38-45.

10. Bruera E, et al. The Edmonton Symptom Assessment System (ESAS): a simple method for the assessment of palliative care patients. J Palliat Care 1991;7:6

11. Kwon JH, David Hui, Gary Chisholm, et al. Journal of Palliative Medicine. February 2013, 16(2): 148-155.

12. Dalal S, et al. Use of Palliative Care Services in a Tertiary Cancer Center. The Oncologist. 2016;21: 110-118.

13. Molassiotis $P$, et al. Use of complementary and alternative medicine in cancer patients: a European survey. Annals of Oncology, 2005; 16: 655-663,.

14. Girgis A, Adams J, Sibbritt D. The use of complementary and alternative therapies by patients with cancer. Oncol Res. 2005; 15: 281-289.

15. Richardson MA, Sanders T, Palmer JL, et al. Complementary/alternative medicine use in a comprehensive cancer center and the implications for oncology. Journal of Clinical Oncology, 2000; 18: 2505-2514. 
16. Garcia MK, McQuade J, Haddad R, et al. Systematic review of acupuncture in cancer care: a synthesis of the evidence. J Clin Oncol. 2013 Mar 1;31(7):952-60.

17. Mishra SL, et al. Exercise interventions on health-related quality of life for cancer survivors. Cochrane Database Syst Rev. 2012 Aug 15;8:CD007566.

18. Kushi LH, et al. American Cancer Society Guidelines on Nutrition and Physical Activity for Cancer Prevention: Reducing the Risk of Cancer With Healthy Food Choices and Physical Activity. CA Cancer J Clin 2012;62:30-67. 